\title{
Theory of Time-dependent Space-charge-limited Conduction
}

\author{
H. K. Chan ${ }^{1 *}$, Y. Zhou', C. H. Lam ${ }^{3}$ and F. G. Shin ${ }^{3}$ \\ ${ }^{1}$ School of Physics and Astronomy, University of Manchester, \\ Manchester, United Kingdom. \\ ${ }^{2}$ School of Engineering Sciences, KTH, \\ Stockholm, Sweden. \\ ${ }^{3}$ Department of Applied Physics, Hong Kong Polytechnic University, \\ Hong Kong, China. \\ *E-mail: epkeiyeah@yahoo.com.hk
}

\begin{abstract}
We have developed a theory of timedependent space-charge-limited conduction in recent years to understand a hysteresis-shift phenomenon of graded ferroelectric films. The theory is a generalization of the conventional steady-state space-charge-limited conduction model to include (i) two carrier types ( $p$ type and $n$-type), (ii) intrinsic conductivity and (iii) time dependence. Later, the theory was also applied to understand the hysteresis behaviour of homogeneous ferroelectric films. In this paper, a full and updated version of the theory is presented. At the limit of zero intrinsic conductivity, the theory implies the dominance of either carrier type. Accordingly, the independent assumptions of (i) a single carrier type and (ii) a negligible intrinsic conductivity in the conventional steady-state conduction model are interrelated.
\end{abstract}

\section{INTRODUCTION}

Electrical conduction in solid dielectrics is often nonOhmic, due to the presence of space charge and other field-induced effects [1,2]. For a plane-parallel dielectric capacitor of thickness $L$ under a steadily applied voltage $V$, the conventional space-chargelimited conduction model concludes that the current density $J$ and the voltage $V$ have a power-law relation of $J \sim V^{2}[1,2]$. This relation has been experimentally observed for various dielectric materials [3-5]. Its theoretical derivation is summarized as follows:

Consider a dielectric sample with a negligible intrinsic conductivity $\sigma_{o} \approx 0$. Charge carriers of one single type are injected from one electrode and captured by the other. The charge mobility $\mu$ and dielectric permittivity $\varepsilon$ are assumed to be constant throughout the sample. The electric displacement $D$ and free charge density $\rho$ as functions of the position $x$ from one electrode are

$$
D(x)=\varepsilon \cdot E(x)
$$

and

$$
\rho(x)=\frac{d D(x)}{d x}=\varepsilon \cdot \frac{d E(x)}{d x} \quad \text { (Gauss' law) }
$$

respectively. In the absence of both displacement and diffusion currents, the steady-state current density $J$ is equivalent to the conduction-current density $J_{c}$. The continuity of a steady-state current implies that $J$ is independent of the position $x$. It follows that

$$
\begin{aligned}
& J=J_{c}=\mu \cdot \rho(x) \cdot E(x)=\mu \cdot \varepsilon \cdot E(x) \cdot \frac{d E(x)}{d x} \\
& =\frac{\mu \cdot \varepsilon}{2} \cdot \frac{d E(x)^{2}}{d x}=\frac{\mu \cdot \varepsilon}{2 \cdot x} \cdot E(x)^{2} .
\end{aligned}
$$

Equation (3) is based on the boundary condition of $E(0)=0$, as the electric field is neutralized by the injected carriers [1]. From Kirchoff's voltage law and (3), one obtains

$$
V=\int_{0}^{L} E(x) \cdot d x=\left(\frac{8 \cdot J}{9 \cdot \mu \cdot \varepsilon}\right)^{1 / 2} \cdot L^{3 / 2}
$$

or equivalently,

$$
J=\left(\frac{9 \cdot \mu \cdot \varepsilon}{8 \cdot L^{3}}\right) \cdot V^{2} \sim V^{2} \quad(\text { Child's law) }
$$

Despite the success of (5) in describing the spacecharge-limited conduction of various dielectric materials [3-5], there are motivations to derive a more general time-dependent and multi-carrier space-chargelimited conduction formula for wider applications: (i) In the case of a time-varying applied voltage $V(t)$, there are also displacement currents. In this case, one cannot equate $J$ with $J_{c}$, so that (5) cannot describe the corresponding space-charge conduction process. (ii) In many dielectric materials, there are at least two types of carriers, namely $p$-type and $n$-type. The intrinsic conductivity $\sigma_{o}$ is usually small, but it might not be negligible. (iii) For nonlinear dielectrics, the effective dielectric permittivity, defined via (1) as $\varepsilon=D / E$, is not a constant. It could be field-dependent, positiondependent (for inhomogeneous materials), or even timedependent (e.g. for ferroelectrics).

In recent years, we have investigated theoretically [6-8] the peculiar vertical shift of hysteresis loops as observed for graded ferroelectric thin films excited by alternating electric fields [9]. In these graded films, there are gradients of the electric displacement $D$ and the 
corresponding presence of free space charge, according to the Gauss' law in (2). The overall conductivity is affected locally by the free space charge and thus becomes non-Ohmic. To take into account the effects of both space-charge and Ohmic conduction, we have developed a theory of time-dependent space-chargelimited conduction [6]. By incorporating this theory into a model of the graded film, the vertical shift of hysteresis loops was reproduced in the simulations [6]. Later, the theory was also applied to understand the hysteresis behaviour of homogeneous ferroelectric films [10]. In this paper, the formulation of the theory is presented in full, with the limiting case of zero intrinsic conductivity discussed in detail.

\section{THEORETICAL DERIVATION OF THE TIME-DEPENDENT SPACE-CHARGE- LIMITED CONDUCTION FORMULA}

For a time-varying applied voltage $V(t)$, the conductioncurrent density $J_{c}(x, t)$, electric field $E(x, t)$, and the electric displacement $D(x, t)$ are functions of position $x$ and time $t$ for an inhomogeneous dielectric. The conduction-current density can be related to the electric field via a time-dependent conductivity $\sigma(x, t)$ :

$$
J_{c}(x, t)=\sigma(x, t) \cdot E(x, t)
$$

Consider the presence of $p$-type and $n$-type free charge carriers, of position-dependent mobilities $\mu_{p}(x)$ and $\mu_{n}(x)$, and of electric charges $q$ and $-q$, respectively. Here, $\mu_{p}(x), \mu_{n}(x)$ and $q$ are positive values. Due to charge neutrality, the intrinsic concentrations of the two carrier types are equal, and are here denoted as $C_{\text {in }}(x)$. The time-dependent conductivity $\sigma(x, t)$ can be expressed as

$$
\begin{aligned}
\sigma(x, t)= & q \cdot \mu_{p}(x) \cdot\left[C_{i n}(x)+\Delta p(x, t)\right] \\
& -q \cdot\left[-\mu_{n}(x)\right] \cdot\left[C_{i n}(x)+\Delta n(x, t)\right],
\end{aligned}
$$

where $\Delta p(x, t)$ and $\Delta n(x, t)$ are the differences between intrinsic and total concentrations for the $p$-type and $n$ type carriers, respectively. The intrinsic conductivity is defined as

$$
\sigma_{o}(x)=q \cdot\left[\mu_{p}(x)+\mu_{n}(x)\right] \cdot C_{i n}(x)
$$

so that the time-dependent conductivity in (7) can be written as

$$
\begin{aligned}
\sigma(x, t)=\sigma_{o}(x)+q \cdot & {\left[\mu_{p}(x) \cdot \Delta p(x, t)\right.} \\
& \left.+\mu_{n}(x) \cdot \Delta n(x, t)\right]
\end{aligned}
$$

According to (2), the density of free space charge is

$$
\frac{\partial D(x, t)}{\partial x}=q \cdot\left[C_{\text {in }}(x)+\Delta p(x, t)\right]-q \cdot\left[C_{\text {in }}(x)+\Delta n(x, t)\right]
$$

$$
=q \cdot[\Delta p(x, t)-\Delta n(x, t)]
$$

With a dielectric insulator being equivalent to a wide band-gap semiconductor, there are endothermic generation and exothermic recombination of carriers. One can assume that the rates of these endothermic and exothermic processes are equal (i.e. the energy released by carrier recombination is entirely used for carrier generation), with each rate being proportional to the product of the reactants' concentrations. The latter assumption is quite natural because the product of the reactants' concentrations is equal to the number of ways per unit volume the different types of reactants can combine to undergo the process. In a dielectric insulator, the concentration of recombined electrons is orders of magnitude larger than the concentrations of free carriers Accordingly, the concentration of recombined electrons for carrier generation has an insignificant fluctuation with time, i.e. it is practically time-invariant. The rate of carrier recombination is thus also approximately timeinvariant, as it is equal to the rate of carrier generation. One can then approximate the recombination rate $R$ as

$$
\begin{aligned}
R= & R(x) \propto C_{i n}(x)^{2} \\
& =\left[C_{i n}(x)+\Delta p(x, t)\right] \cdot\left[C_{\text {in }}(x)+\Delta n(x, t)\right]
\end{aligned}
$$

(10) and (11) can be rewritten as

$\Delta p(x, t)=\frac{1}{q} \cdot \frac{\partial D(x, t)}{\partial x}+\Delta n(x, t)$

and

$$
\begin{gathered}
C_{i n}(x) \cdot[\Delta p(x, t)+\Delta n(x, t)]+\Delta p(x, t) \cdot \Delta n(x, t) \\
=0,
\end{gathered}
$$

respectively. Further rearrangement of (12) and (13) leads to a pair of quadratic equations for $\Delta p(x, t)$ and $\Delta n(x, t)$ :

$$
\Delta p(x, t)^{2}+B_{p}(x, t) \cdot \Delta p(x, t)+C_{p}(x, t)=0
$$

and

$$
\Delta n(x, t)^{2}+B_{n}(x, t) \cdot \Delta n(x, t)+C_{n}(x, t)=0,
$$

where

$$
\begin{aligned}
& B_{p}(x, t) \equiv-\frac{1}{q} \cdot \frac{\partial D(x, t)}{\partial x}+2 \cdot C_{i n}(x), \\
& C_{p}(x, t) \equiv-\frac{C_{i n}(x)}{q} \cdot \frac{\partial D(x, t)}{\partial x}, \\
& B_{n}(x, t) \equiv \frac{1}{q} \cdot \frac{\partial D(x, t)}{\partial x}+2 \cdot C_{i n}(x)
\end{aligned}
$$

and

$$
C_{n}(x, t) \equiv \frac{C_{i n}(x)}{q} \cdot \frac{\partial D(x, t)}{\partial x} .
$$


$\Delta p(x, t)$ and $\Delta n(x, t)$ in (9) can then be replaced by the roots of their quadratic equations. This leads to

$$
\begin{aligned}
& q \cdot\left[\mu_{p}(x) \cdot \Delta p(x, t)+\mu_{n}(x) \cdot \Delta n(x, t)\right] \\
& =q \cdot\left[\begin{array}{l}
\mu_{p}(x) \cdot \frac{-B_{p}(x, t) \pm \sqrt{B_{p}(x, t)^{2}-4 \cdot C_{p}(x, t)}}{2} \\
+\mu_{n}(x) \cdot \frac{-B_{n}(x, t) \pm \sqrt{B_{n}(x, t)^{2}-4 \cdot C_{n}(x, t)}}{2}
\end{array}\right] .
\end{aligned}
$$

Using the definitions from (16) to (19), together with the definition of the intrinsic conductivity $\sigma_{o}(x)$ in (8), one obtains

$$
\begin{aligned}
& -\frac{q}{2} \cdot\left[\mu_{p}(x) \cdot B_{p}(x, t)+\mu_{n}(x) \cdot B_{n}(x, t)\right] \\
& =\frac{\left[\mu_{p}(x)-\mu_{n}(x)\right]}{2} \cdot \frac{\partial D(x, t)}{\partial x}-\sigma_{o}(x)
\end{aligned}
$$

and

$$
\begin{aligned}
& \frac{q}{2} \cdot \sqrt{B_{p}(x, t)^{2}-4 \cdot C_{p}(x, t)}=\frac{q}{2} \cdot \sqrt{B_{n}(x, t)^{2}-4 \cdot C_{n}(x, t)} \\
& =\frac{1}{2} \cdot \sqrt{\left[\frac{\partial D(x, t)}{\partial x}\right]^{2}+\frac{4 \cdot \sigma_{o}(x)^{2}}{\left[\mu_{p}(x)+\mu_{n}(x)\right]^{2}}}
\end{aligned}
$$

Using (21) and (22), (20) can be written as

$$
\begin{aligned}
& q \cdot\left[\mu_{p}(x) \cdot \Delta p(x, t)+\mu_{n}(x) \cdot \Delta n(x, t)\right] \\
& =\frac{\left[\mu_{p}(x)-\mu_{n}(x)\right]}{2} \cdot \frac{\partial D(x, t)}{\partial x}-\sigma_{o}(x) \\
& +\frac{\left[ \pm \mu_{p}(x) \pm \mu_{n}(x)\right]}{2} \cdot \sqrt{\left[\frac{\partial D(x, t)}{\partial x}\right]^{2}+\frac{4 \cdot \sigma_{o}(x)^{2}}{\left[\mu_{p}(x)+\mu_{n}(x)\right]^{2}}}
\end{aligned}
$$

Using (9), (23) can be transformed into an expression for the time-dependent conductivity $\sigma(x, t)$ :

$$
\begin{aligned}
& \sigma(x, t)=\frac{\left[\mu_{p}(x)-\mu_{n}(x)\right]}{2} \cdot \frac{\partial D(x, t)}{\partial x} \\
& +\sqrt{\left\{\frac{\left[ \pm \mu_{p}(x) \pm \mu_{n}(x)\right]}{2} \cdot \frac{\partial D(x, t)}{\partial x}\right\}^{2}+\frac{\left[ \pm \mu_{p}(x) \pm \mu_{n}(x)\right]^{2} \cdot \sigma_{o}(x)^{2}}{\left[\mu_{p}(x)+\mu_{n}(x)\right]^{2}}} \cdot
\end{aligned}
$$

According to (10) and (24), for $\Delta p(x, t)=\Delta n(x, t)=0$, it is

$$
\frac{\partial D(x, t)}{\partial x}=0
$$

and

$$
\sigma(x, t)=\sigma_{o}(x)=\frac{\left[ \pm \mu_{p}(x) \pm \mu_{n}(x)\right]}{\left[\mu_{p}(x)+\mu_{n}(x)\right]} \cdot \sigma_{o}(x)
$$

Equqation (26) implies $\left[ \pm \mu_{p}(x) \pm \mu_{n}(x)\right]=\left[\mu_{p}(x)+\mu_{n}(x)\right]$,

so that (24) should be written as

$$
\begin{aligned}
& \sigma(x, t)=\frac{\left[\mu_{p}(x)-\mu_{n}(x)\right]}{2} \cdot \frac{\partial D(x, t)}{\partial x} \\
& +\sqrt{\left\{\frac{\left[\mu_{p}(x)+\mu_{n}(x)\right]}{2} \cdot \frac{\partial D(x, t)}{\partial x}\right\}^{2}+\sigma_{o}(x)^{2}},
\end{aligned}
$$

which we here refer to as the time-dependent spacecharge-limited conduction formula.

\section{LIMIT OF ZERO INTRINSIC CONDUCTIVITY}

If the intrinsic conductivity $\sigma_{o}(x)$ vanishes but the timedependent conductivity $\sigma(x, t)$ remains finite, the spacecharge density $\partial D(x, t) / \partial$ must be non-zero, according to (28). In this case, the conduction mechanism is spacecharge-limited. According to the expression for $\sigma(x, t)$ in (7), either of the mobility values $\mu_{p}(x)$ and $\mu_{n}(x)$ has to be non-zero for $\sigma(x, t)$ to remain finite. From the definition of the intrinsic conductivity $\sigma_{o}(x)$ in (8), the intrinsic concentration $C_{i n}(x)$ must tend to zero for $\sigma_{o}(x)$ to vanish, because the mobility sum $\left[\mu_{p}(x)+\mu_{n}(x)\right]$ is finite.

For a vanishing $C_{i n}(x),(13)$ reduces to

$$
\Delta p(x, t) \cdot \Delta n(x, t) \approx 0
$$

Multiplying both sides of (12) by $\Delta p(x, t)$ or $\Delta n(x, t)$, and then applying (29), one obtains

$$
\Delta p(x, t) \cdot\left[\Delta p(x, t)-\frac{1}{q} \cdot \frac{\partial D(x, t)}{\partial x}\right] \approx 0
$$

and

$$
\Delta n(x, t) \cdot\left[\Delta n(x, t)+\frac{1}{q} \cdot \frac{\partial D(x, t)}{\partial x}\right] \approx 0
$$

for $\Delta p(x, t)$ and $\Delta n(x, t)$, respectively. The solutions to (30) and (31) are

$$
\Delta p(x, t) \approx 0 \text { or } \Delta p(x, t) \approx \frac{1}{q} \cdot \frac{\partial D(x, t)}{\partial x}
$$

and

$$
\Delta n(x, t) \approx 0 \text { or } \Delta n(x, t) \approx-\frac{1}{q} \cdot \frac{\partial D(x, t)}{\partial x},
$$

respectively. According to (12), however, only the following combinations of solutions are allowed: 


$$
\Delta p(x, t) \approx 0 \text { and } \Delta n(x, t) \approx-\frac{1}{q} \cdot \frac{\partial D(x, t)}{\partial x}
$$

and

$$
\Delta n(x, t) \approx 0 \text { and } \Delta p(x, t) \approx \frac{1}{q} \cdot \frac{\partial D(x, t)}{\partial x} .
$$

As the intrinsic concentration $C_{i n}(x)$ vanishes, $\Delta p(x, t)$ and $\Delta n(x, t)$ become the total concentrations of $p$-type and $n$-type carriers, respectively. It follows that:

\section{At the limit of zero intrinsic conductivity $\sigma_{o}(x)$, either $p$ - type or n-type carriers are dominant}

Since the total concentrations $\Delta p(x, t)$ and $\Delta n(x, t)$ must be positive by definition, (34) and (35) imply $\partial \mathcal{D}(x, t) / \partial x<0$ and $\partial \mathcal{D}(x, t) / \partial x>0$, as consistent with the dominance of $n$-type and $p$-type carriers, respectively.

For a vanishing intrinsic conductivity $\sigma_{o}(x),(28)$ can be written as

$$
\begin{aligned}
\sigma(x, t) \approx & \frac{\left[\mu_{p}(x)-\mu_{n}(x)\right]}{2} \cdot \frac{\partial D(x, t)}{\partial x} \\
& +\frac{\left[\mu_{p}(x)+\mu_{n}(x)\right]}{2} \cdot\left|\frac{\partial D(x, t)}{\partial x}\right| .
\end{aligned}
$$

For $\partial(x, t) / \partial x>0$ ( $p$-type dominance), it is

$$
\begin{aligned}
\sigma(x, t) \approx & \frac{\left[\mu_{p}(x)-\mu_{n}(x)\right]}{2} \cdot \frac{\partial D(x, t)}{\partial x} \\
& +\frac{\left[\mu_{p}(x)+\mu_{n}(x)\right]}{2} \cdot \frac{\partial D(x, t)}{\partial x} \\
\approx & \mu_{p}(x) \cdot \frac{\partial D(x, t)}{\partial x}
\end{aligned}
$$

Else, for $\partial D(x, t) / \partial x<0$ ( $n$-type dominance), it is

$$
\begin{aligned}
\sigma(x, t) \approx & \frac{\left[\mu_{p}(x)-\mu_{n}(x)\right]}{2} \cdot \frac{\partial D(x, t)}{\partial x} \\
& +\frac{\left[\mu_{p}(x)+\mu_{n}(x)\right]}{2} \cdot\left(-\frac{\partial D(x, t)}{\partial x}\right) \\
\approx & -\mu_{n}(x) \cdot \frac{\partial D(x, t)}{\partial x} .
\end{aligned}
$$

(37) and (38) together verify that the mobility in the time- dependent conductivity $\sigma(x, t)$ is equal to that of the dominant carrier type.

\section{CONCLUSIONS}

In this paper, a theory of time-dependent space-chargelimited conduction is presented in full. It is a generalization of the conventional steady-state spacecharge-limited conduction model to include (i) two carrier types ( $p$-type and $n$-type), (ii) intrinsic conductivity and (iii) time dependence. According to the theory, the time-dependent conductivity $\sigma(x, t)$ is related to the space-charge density $\partial D(x, t) / \partial x$ and intrinsic conductivity $\sigma_{\circ}(x)$ in a nonlinear fashion (Equation (28)) For the limiting case of zero intrinsic conductivity, the theory concludes that either $p$-type or $n$-type carriers are dominant, thus correlating the independent assumptions of (i) a single carrier type and (ii) a negligible intrinsic conductivity in the conventional steady-state model.

\section{REFERENCES}

[1] R. Coelho, Physics of Dielectrics for the Engineer, Elsevier Scientific: New York (1979)

[2] M. Pope and C. E. Swemberg, Electronic Processes in Organic Crystal and Polymer, Oxford University Press: Oxford (1998).

[3] Kwang S. Suh, Jong Eun Kim, Woo Jung Oh, Ho Gyu Yoon and Tatsuo Takada, "Charge distribution and conduction characteristics of 2vinylpyridine-grafted polyethylenes", J. Appl. Phys. 87, 7333 (2000).

[4] Apurba Laha and S. B. Krupanidhi, "Leakage current conduction of pulsed excimer laser ablated $\mathrm{BaBi}_{2} \mathrm{Nb}_{2} \mathrm{O}_{9}$ thin films", J. Appl. Phys. 92, 415 (2002).

[5] A. Carbone, B. K. Kotowska and D. Kotowski, "Space-Charge-Limited Current Fluctuations in Organic Semiconductors", Phys. Rev. Lett. 95, 236601 (2005)

[6] H. K. Chan, C. H. Lam and F. G. Shin, "Timedependent space-charge-limited conduction as a possible origin of the polarization offsets observed in compositionally graded ferroelectric films", J. Appl. Phys. 95, 2665 (2004).

[7] Y. Zhou, H. K. Chan, C. H. Lam and F. G. Shin, "Effects of polarization and permittivity gradients and other parameters on the anomalous vertical shift behavior of graded ferroelectric thin films", J. Appl. Phys 98, 034105 (2005).

[8] C. K. Wong and F. G. Shin, "Modeling of anomalous hysteresis behavior of compositionally graded ferroelectric films at low fields", $J$. Appl. Phys. 98, 024104 (2005).

[9] Norman W. Schubring, Joseph V. Mantese, Adolph L. Micheli, Antonio B. Catalan, and Richard J. Lopez, "Charge pumping and pseudopyroelectric effect in active ferroelectric relaxortype films", Phys. Rev. Lett. 68, 1778 (1992).

[10] Y. Zhou, H. K. Chan, C. H. Lam and F. G. Shin, "Mechanisms of imprint effect on ferroelectric thin films", J. Appl. Phys. 98, 024111 (2005). 